\title{
An Opportunistic Array Beamforming Technique Based on Binary Multiobjective Wind Driven Optimization Method
}

\author{
Zhenkai Zhang, ${ }^{1,2,3}$ Sana Salous, ${ }^{3}$ Hailin $\mathrm{Li}^{1}{ }^{1}$ and Yubo Tian ${ }^{2}$ \\ ${ }^{1}$ Key Laboratory of Radar Imaging and Microwave Photonics, Nanjing University of Aeronautics and Astronautics, \\ Nanjing 211106, China \\ ${ }^{2}$ College of Electronic Information, Jiangsu University of Science and Technology, Zhenjiang 212003, China \\ ${ }^{3}$ Centre for Communication Systems, Durham University, Durham DH1 3LE, UK \\ Correspondence should be addressed to Zhenkai Zhang; zzk@nuaa.edu.cn
}

Received 10 May 2015; Revised 1 August 2015; Accepted 19 August 2015

Academic Editor: Stefano Selleri

Copyright (c) 2015 Zhenkai Zhang et al. This is an open access article distributed under the Creative Commons Attribution License, which permits unrestricted use, distribution, and reproduction in any medium, provided the original work is properly cited.

\begin{abstract}
We present a novel binary version of multiobjective wind driven optimization (WDO) for emitted beamforming of opportunistic array radar, which is assumed as a multiobjective optimization problem. Firstly, the emitted signal model and objective functions of optimization are presented. Then the algorithm proposes a new definition of the position vector of air parcel, and brings a good discretization interpretation of continuous WDO. For multiobjective optimization, the grey relational grade (GRG) is then used to measure the similarity between the best two solutions for these two objectives. The best pressure locations with the maximum GRG will be recorded as the best two candidate solutions to the problem, and a final optimization result will be selected according to the importance of the two objectives. Finally, the proposed improved WDO has been applied for the optimal design of beamforming of the opportunistic antenna array, which needs a trade-off between the $3 \mathrm{~dB}$ main beam width and sidelobe level. The simulation results show that the proposed method outperforms conventional particle swarm optimization (PSO) in the optimal beamforming by achieving more reduction in the sidelobe level and saving more runtime.
\end{abstract}

\section{Introduction}

A new radar concept, the opportunistic array radar which is also called distributed phased array radar, has been proposed by some scholars in recent years [1-3], based on digital array radar. Opportunistic array radar is a new generation radar system based on the stealth of the platform, which can improve the modern radar performance effectively. The array elements of opportunistic array radar are distributed randomly in the aircraft or ship platform. The beamforming data, control signals, and target return signals are sent wirelessly between the transmitting/receiving modules where the beamformer and signal processor are located away from the modules. The concept, principle, and characteristics of the opportunistic digital array radar are presented in [2]. Phase orthogonal code sets with low autocorrelation and crosscorrelation properties, which can be used in the opportunistic array radar systems, are reported in [3]. The research of the opportunistic array radar is still at the beginning; for example, there are very few studies on the design of emitted beamforming, which is supposed to be a multiobjective optimization problem. The main challenge to solving such multiobjective optimization problem is that there is rarely a decision that simultaneously optimizes all objectives.

There exist at least two different research fields which focus on solving multiobjective optimization problems [4]: multiple criteria decision making and evolutionary multiobjective optimization (EMO). Presently, EMO algorithms are widely used for solving multiobjective optimization problems due to their advantages [4]. Particle swarm optimization (PSO) is relatively a recent evolutionary computation technique based on swarm intelligence. Compared with genetic algorithms, PSO is computationally less expensive and can converge more quickly [5]. Therefore, PSO has been used as an effective technique in many fields, including multiobjective optimization [6,7]. In [8] the Pareto optimal solutions of the problem were found using a binary PSO algorithm and a fuzzy satisfying method was applied to select 
the optimal solution. Building on these successful optimization algorithms, a new optimization method called wind driven optimization (WDO) is presented in [9]. However, it is a continuous optimization method for a single objective.

In this study, a binary multiobjective WDO (BiMOWDO) technique based grey relational grade (GRG) is presented, and the design of a multiobjective optimization model for opportunistic array beamforming involves finding an acceptable trade-off between beam width and sidelobe level, with the constraint of desired emitted power. The remainder of this paper is organized as follows. Section 2 presents the emitted signal model of opportunistic array radar and builds the objective functions for beamforming. Sections 3 and 4 describe the wind driven optimization method and GRG theory, respectively. Section 4 proposes the Bi-MOWDO technique based on GRG. Simulation results and conclusions are provided in Sections 5 and 6, respectively.

\section{Signal Modeling of Opportunistic Array Radar and Objective Function of Beamforming}

In order to accomplish the design of emitted power, the number and position of the array units should be optimized to obtain the emitted beamforming with excellent performance. So the objective functions should be built based initially on the wideband signal model.

2.1. Wideband Signal Model. For a $m \times n$ plane array with arbitrary geometry of opportunistic array radar, we selected a chirp waveform to illustrate the advantages of the proposed method. The time-domain chirp waveform $\mathbf{S}(t)$ of every array unit can be written as

$$
\mathbf{S}(t)=\mathbf{A}(t) e^{j 2 \pi\left(f_{0} t+\mu t^{2} / 2\right)}
$$

where $f_{0}, \mu$, and $\mathbf{A}(t)$ denote the carrier frequency, chirp rate, and signal amplitude, respectively.

We define the steering element of the beamformer at the direction of $\left(\theta_{0}, \varphi_{0}\right)$ with the $k$ th array unit position of $\left(0, y_{k}, z_{k}\right)$ :

$$
a_{k 0}=\exp \left(-j \frac{2 \pi}{\lambda} d_{k 0}\right)
$$

Here, $\theta_{0}$ and $\varphi_{0}$ are the azimuth angle and elevation angle of the target, respectively, which are defined in Figure 1. $d_{k 0}=$ $\left(x_{k} \sin \theta_{0} \cos \varphi_{0}+y_{k} \sin \varphi_{0}\right) d$ and $d$ is the array distance. $\tau_{k 0}$ denotes the delay of the desired signal impinging on the $k \mathrm{th}$ sensor of the array from the desired direction $\left(\theta_{0}, \varphi_{0}\right), \tau_{k 0}=$ $d_{k 0} / c$, and $c$ is the speed of propagation of an electromagnetic wave in free space.

The emitted signal from the $k$ th array unit can be represented as

$$
\begin{aligned}
\mathbf{S}_{k}(t) & =\mathbf{S}\left(t-\tau_{k 0}\right) \\
& =\mathbf{A}\left(t-\tau_{k 0}\right) e^{j 2 \pi\left[f_{0}\left(t-\tau_{k 0}\right)+\mu\left(t-\tau_{k 0}\right)^{2} / 2\right]} .
\end{aligned}
$$



FIgURE 1: Definition of azimuth and elevation angles.

The signal emitted in the direction $\left(\theta_{i}, \varphi_{i}\right)$ is given as

$$
\begin{aligned}
\mathbf{Y}_{i}(t) & =\sum_{k=0}^{K-1} \mathbf{S}_{k}\left(t+\tau_{k i}\right) w_{k 0} a_{k i} \\
& =\sum_{k=0}^{K-1} \mathbf{S}\left(t+\tau_{k i}-\tau_{k 0}\right) w_{k 0} a_{k i},
\end{aligned}
$$

where $i=1,2, \ldots, D-1, K=m n, w_{k i}$ is the weight element of the $k$ th array, $\tau_{k i}=\left(x_{k} \cos \theta_{i} \cos \varphi_{i}+z_{k} \sin \varphi_{i}\right) d / c$, and $D$ denotes the number of emitted directions.

The emitted power $P$ after impedance normalization at time $t$ can be represented as

$$
\begin{array}{r}
P=\left\{\left|\mathbf{Y}_{\mathbf{i}}(t)\right|^{2}\right\}=\left\{\left|\sum_{k=0}^{K-1} \mathbf{S}\left(t+\tau_{k i}-\tau_{k 0}\right)\right|^{2}\right\}, \\
i=0,1, \ldots, D-1,
\end{array}
$$

where $w_{k 0}=\bar{a}_{k 0}, \bar{a}_{k 0}$ is the complex conjugate of $a_{k 0}, w_{k 0} a_{k 0}=$ 1 , and $|\cdot|$ denotes modulus.

2.2. Objective Functions for Beamforming. As we know, the emitted power $\mathbf{P}$ is a function of the array unit positions and number. The objective functions to minimize the sidelobe $f_{1}$ and main lobe width $f_{2}$ are given below,

$$
\begin{aligned}
f_{1}=\min _{\left\{\mathbf{m}_{i}, \mathbf{n}_{j}\right\} \subset\{\mathbf{M}, \mathbf{N}\}} & P_{p 1} \\
f_{2}=\min _{\left\{\mathbf{m}_{i}, \mathbf{n}_{j}\right\} \subset\{\mathbf{M}, \mathbf{N}\}} & \left(\theta_{3 \mathrm{~dB}}+\varphi_{3 \mathrm{~dB}}\right) \\
& \text { Subject to: } \quad P_{T}=P_{\mathrm{des}},
\end{aligned}
$$

where $P_{p 1}$ is the maximum sidelobe level, $\theta_{3 \mathrm{~dB}}$ and $\varphi_{3 \mathrm{~dB}}$ are the $3 \mathrm{~dB}$ width of the beam in azimuth angle and elevation angle, respectively, $\mathbf{M}$ and $\mathbf{N}$ are the row and column set of the array position in the radar array plane, $\mathbf{m}_{i}$ and $\mathbf{n}_{j}$ are the row and column set of selected array position, and $P_{T}$ and $P_{\text {des }}$ are emitted power and desired power of the beam, respectively.

The objectives $f_{1}$ and $f_{2}$ often conflict with each other. Improvement of one objective may lead to deterioration of 
the other. So a single solution that can optimize all objectives simultaneously does not exit.

As we know, for a general multiobjective optimization problem, the ideal procedure is to find a set of Pareto optimal solutions $[10,11]$ first and then to choose one solution from the set by using some other information. So we have to find a trade-off between $3 \mathrm{~dB}$ main beam width and sidelobe level. In the proposed algorithm, the selected array units in the array plane will be marked 1 , and the others will be marked 0 . Therefore, a binary multiobjective optimization method will be used to obtain the solution. In this paper, an improved wind driven optimization method will be presented to obtain the best solution.

\section{Wind Driven Optimization Technique}

The inspiration for WDO comes from the earth's atmosphere, where wind blows in an attempt to equalize horizontal imbalances in the air pressure [9]. In WDO first presented in [9], at every iteration, each air parcel's velocity and position are updated as its exploration of search space progresses. Thus, the change in velocity $\Delta \mathbf{u}$ can be written as $\Delta \mathbf{u}=$ $\mathbf{u}_{\text {new }}-\mathbf{u}_{\text {cur }}$, where $\mathbf{u}_{\text {cur }}$ is the velocity at the current iteration and $\mathbf{u}_{\text {new }}$ is the velocity in the next iteration. As described in [9], the influence of the Coriolis force is replaced by the velocity influence from another randomly chosen dimension of the same air parcel, $\mathbf{u}_{\text {cur }}^{\text {other dim }}$, and all other coefficients are combined into a single term $c$; for example, $c=-2|\Omega| U_{g} T_{e}$. The $\mathbf{u}_{\text {new }}$ can be written as

$$
\begin{aligned}
\mathbf{u}_{\mathrm{new}}= & (1-\alpha) \mathbf{u}_{\mathrm{cur}}-g \mathbf{x}_{\mathrm{cur}} \\
& +\left(U_{g} T_{e}\left|\frac{1}{i}-1\right|\left(\mathbf{x}_{\mathrm{opt}}-\mathbf{x}_{\mathrm{cur}}\right)\right) \\
& +\left(\frac{c \mathbf{u}_{\mathrm{cur}}^{\text {other dim }}}{i}\right),
\end{aligned}
$$

where $\Omega$ represents the rotation of the earth, $i$ represents the ranking among all air parcels, $\mathbf{x}_{\mathrm{opt}}$ and $\mathbf{x}_{\mathrm{cur}}$ represent the current location and optimum location, respectively, $\alpha$ is the friction coefficient, $g$ is the earth's gravitational field, $U_{g}$ is the universal gas constant, and $T_{e}$ is the temperature. Once the new velocity is calculated, the position can be updated by utilizing the following equation:

$$
\mathbf{x}_{\text {new }}=\mathbf{x}_{\text {cur }}+\mathbf{u}_{\text {new }} \Delta t \text {, }
$$

where $\Delta t$ is a time step.

A population of air parcels starts at random positions in the search space with random velocities. Each air parcel's velocity and position are adjusted, each iteration as the parcels move toward an optimum pressure location and the optimum solution at the end of the last iteration. In this manner WDO offers a simple and effective way to solve complex optimization problems. The implementation of WDO is illustrated in Figure 2. As seen in the flowchart, the algorithm starts with the initialization stage, where all parameters related to the WDO as well as the other parameters related to the optimization problem have to be defined. Also, one must

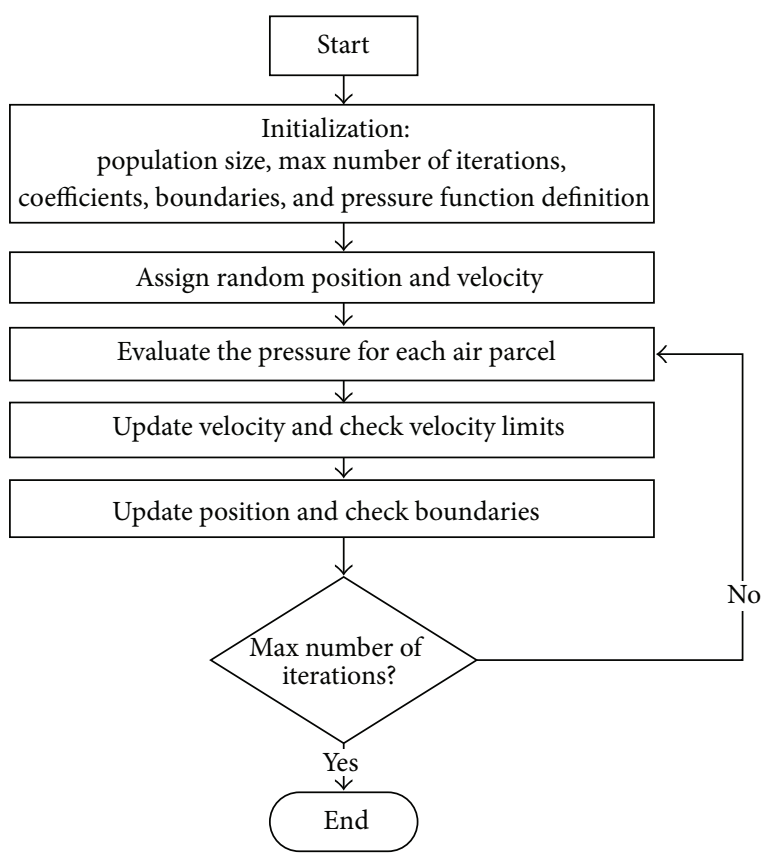

FIGURE 2: Implementation of WDO.

define a pressure function as a fitness function and establish parameter boundaries. Once the optimization problem is set up, the population of air parcels are randomly distributed over the $N$-dimensional search space and assigned random velocities. The next step is to evaluate the pressure values of each air parcel at its current position.

Once the pressure values have been evaluated, the population is ranked based on their pressure, and the velocity updated according to (7) is applied with the restrictions given in

$$
u_{\text {new }}^{*}= \begin{cases}u_{\max } & \text { if } u_{\text {new }}>u_{\max } \\ -u_{\max } & \text { if } u_{\text {new }}<-u_{\max }\end{cases}
$$

The positions for the next iteration are updated by utilizing (8), and the boundaries are checked to prevent any air parcel from exiting the search space. Once all the updates are carried out, the parcel pressures at the new locations are evaluated. This procedure continues until the maximum number of iterations is reached. Finally, the best pressure location at the end of the last iteration is recorded as the optimization result and, hence, the best candidate solution to the problem.

\section{Grey Relational Grade}

Grey system theory was proposed by Ju-Long in 1982 [12]. It can be used to analyze the indeterminate and incomplete data to establish the systematic relations. GRG in grey system theory expresses the comparability between two patterns. In other words, GRG can be viewed as a measure of similarity for finite sequences and has been widely used and developed [13]. Desired sequence $Y_{0}$ and inspected sequence $Y_{j}$ are 
represented as $Y_{0}=\left\{y_{0}(k) \mid k=1,2, \ldots, n\right\}$ and $Y_{j}=\left\{y_{j}(k) \mid\right.$ $k=1,2, \ldots, n, j=1,2, \ldots, m\}$, respectively.

The grey relational coefficient between $Y_{j}$ and $Y_{0}$ at the $k$ th component is defined as follows:

$$
\varepsilon_{0 j}(k)=\frac{A_{1}+\zeta A_{2}}{\left|y_{0}(k)-y_{j}(k)\right|+\zeta A_{2}},
$$

where $A_{1}=\min _{j^{\prime}} \min _{k^{\prime}}\left|y_{0}\left(k^{\prime}\right)-y_{j^{\prime}}\left(k^{\prime}\right)\right| ; A_{2}=\max _{j^{\prime}} \max _{k^{\prime}}$ $\left|y_{0}\left(k^{\prime}\right)-y_{j^{\prime}}\left(k^{\prime}\right)\right|$.

The corresponding GRG grey_relation $\left(Y_{0}, Y_{j}\right)$ has the form of

$$
\text { grey_relation }\left(Y_{0}, Y_{j}\right)=\frac{1}{n} \sum_{k=1}^{n} \varepsilon_{0 j}(k) \text {, }
$$

where $\zeta$ is the resolution coefficient which controls the resolution between the two distance factors and it is also used to reduce possible influence of distortion form bias by excessive $A_{2}$. Usually, $\zeta$ is assumed as 0.5 to fit the practical requirements. The larger GRG indicates the greater similarity between the two sequences. GRG is used to measure the similarity between two best solutions belonging to the two objectives in the proposed binary multiobjective WDO algorithm.

\section{Improved Binary Wind Driven Optimization Technique Based on GRG for Multiobjective Optimization}

In order to solve the optimization problem in Section 2, the original WDO method is modified with the binary character and can be used for multiobjective optimization with the help of GRG. The major difference between binary WDO with continuous version is that the amount of position displacement per iteration is rather defined in terms of probabilities that a bit will change to one. In continuous WDO, the coordinates of the air parcel as the parcel positions are represented in continuous values; meanwhile in binary WDO parcel's coordinates are shown in discrete values. Velocity updating in binary WDO is similar to WDO, but it has velocity clamping to balance the exploration and exploitation in the search space. Moreover, in binary WDO the position updating is based on a sigmoid function as shown in

$$
\begin{aligned}
\mathbf{S}\left(\mathbf{u}_{\text {new }}\right) & =\frac{1}{\left(1+\exp \left(-\mathbf{u}_{\text {new }}\right)\right)} \\
\mathbf{x}_{\text {new }} & = \begin{cases}0 & \text { if } \mathbf{S}\left(\mathbf{u}_{\text {new }}\right) \leq \text { rand } \\
1 & \text { if } \mathbf{S}\left(\mathbf{u}_{\text {new }}\right)>\text { rand }\end{cases}
\end{aligned}
$$

where $\mathbf{S}()$ is a sigmoid function and rand is a quasi-random number uniformly distributed within the range of $[0,1]$.

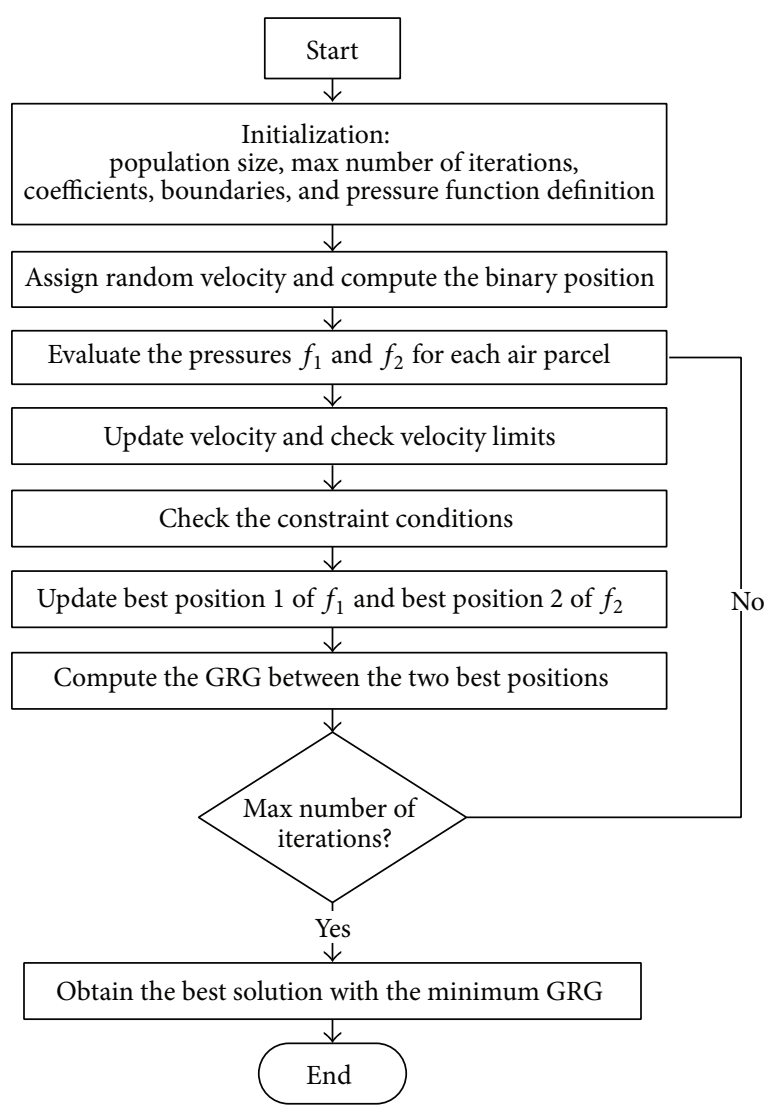

Figure 3: Implementation of Bi-MOWDO.

Figure 3 illustrates the proposed Bi-MOWDO algorithm. It integrates the multiobjective method and grey relational grade into binary WDO algorithm. Comparing with the original WDO, the algorithm has the following features:

(1) When calculating pressure of the air parcels, both objectives $f_{1}(x)$ and $f_{2}(x)$ need to be evaluated.

(2) If parcel's new location is better than its best past location, the best location is updated.

(3) Grey relational grade is used to measure the similarity between two best solutions belonging to the two objectives.

In the Bi-MOWDO, the positions of the two objectives for the next iteration are updated by utilizing (13) after pressures $f_{1}$ and $f_{2}$ are evaluated, and the velocity and constraint conditions are checked to make sure all air parcels are valid. Once all the updates of the two best positions are carried out, the GRG between them are evaluated by using (5). This procedure continues until the maximum number of iterations is reached. Finally, the best pressure locations with the maximum GRG will be recorded as the best two candidate solutions to the problem, and the final optimization result will be selected according to the importance of the two objectives. 


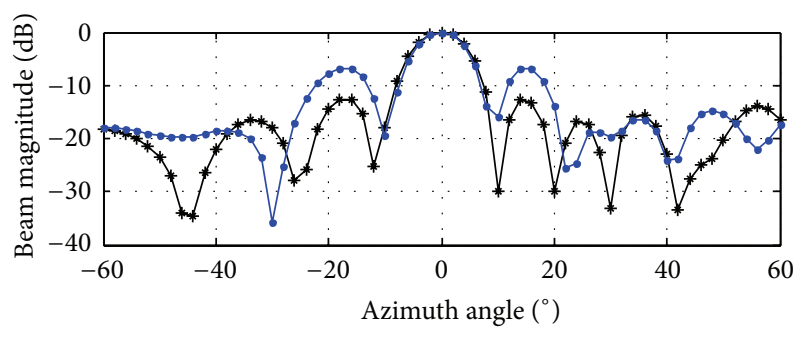

$\rightarrow$ * Bi-MOWDO

$\rightarrow$ Bi-MOPSO

(a) Magnitude at elevation angle $0^{\circ}$



$\rightarrow$ Bi-MOWDO

- Bi-MOPSO

(b) Magnitude at azimuth angle $0^{\circ}$

FIGURE 4: Normalized beam patterns of power $56 \mathrm{~W}$.

\section{Simulation Results}

Here, we are interested in emitted beamforming of opportunistic array radar. In the results presented here, $20 \times 20$ antennas are distributed uniformly around the opportunistic array plane, which are also assumed to be isotropic. Performance of the proposed beamforming method based on BiMOWDO is compared to the binary multiobjective method Bi-MOPSO in [8].

From (7) we note that the friction coefficient $\alpha$ and the gravitational force coefficient $g$ should only vary in the range $[0,1]$. On the other hand, $U_{g} T_{e}$ and $c$ can accommodate a wider range of values, which are allowed to vary in the range of $[0,5]$. The target is placed at $\left(0^{\circ}, 0^{\circ}\right)$ in azimuth and elevation angles. Carrier frequency, bandwidth, and voltage of the emitted signal are $3 \times 10^{9} \mathrm{~Hz}, 200 \times 10^{6} \mathrm{~Hz}$, and $0.15 \mathrm{~V}$, respectively. The simulation shows the wideband beamformer with the desired power $225 \mathrm{~W}$ and $56 \mathrm{~W}$, which need 100 and 50 working array units, respectively, according to (4) and (5). As a result, the total number of dimensions is chosen to be 100 and 50 in the beamforming optimization. For every trial in the Bi-MOWDO, a population of 100 air parcels was optimized for a maximum of 50 iterations. The numbers of particles and iterations of Bi-MOPSO are chosen equal to 100 and 50 in the simulations.

Figures 4, 5, 7, and 8 show the resulting array response of the emitted $56 \mathrm{~W}$ and $225 \mathrm{~W}$, respectively, from which we can see the two-beam former based $\mathrm{Bi}-\mathrm{MOPSO}$ and proposed $\mathrm{Bi}$ MOWDO method with their emitted main lobes of desired powers at the desired direction and sufficient attenuation in the sidelobe regions. The proposed beamforming method



Figure 5: 3D beam patterns of power $56 \mathrm{~W}$.

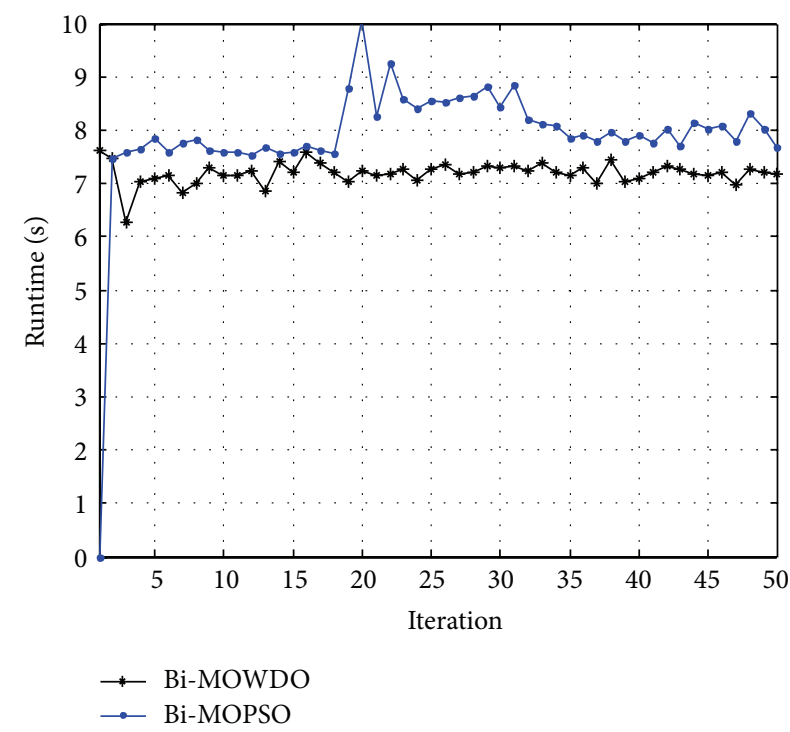

Figure 6: Runtime of every iteration for power $56 \mathrm{~W}$.

provides dramatic improvement of the sidelobe level with almost the same $3 \mathrm{~dB}$ beam width, as compared to the beamforming method based Bi-MOPSO. Runtime for optimizing the two desired powers at every iteration is calculated and plotted and can be seen in Figures 6 and 9, from which we can clearly see that the proposed Bi-MOWDO method saves more time than the Bi-MOPSO method.

In conclusion, the simulation demonstrates the effectiveness of the proposed beamformer method based on BiMOWDO. As it is shown in [9], the position and velocity update rules in WDO are similar to those in PSO; however, the gravitational pull within the velocity update equation in WDO can provide advantages over PSO, where particles occasionally attempt to fly out of and sometimes get stuck at the boundaries. But the gravitational pull in WDO provides a favorable contribution, which prevents air parcels from remaining trapped at the boundary for long periods of time and pulls them back into the search space. 


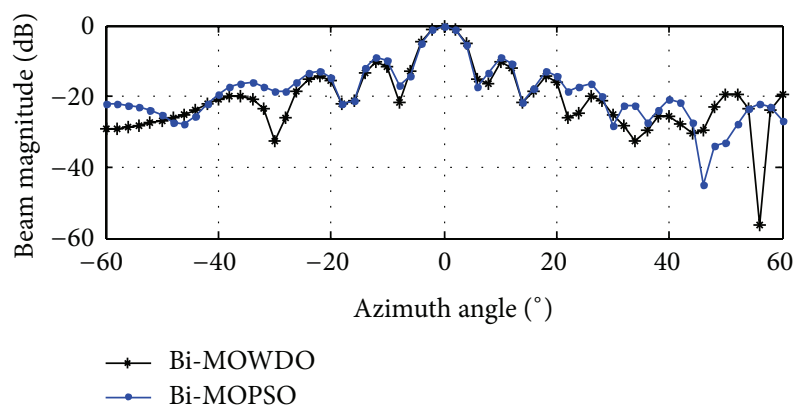

(a) Magnitude at elevation angle $0^{\circ}$



* Bi-MOWDO

$\rightarrow$ Bi-MOPSO

(b) Magnitude at azimuth angle $0^{\circ}$

FigURE 7: Normalized beam patterns of power $225 \mathrm{~W}$.

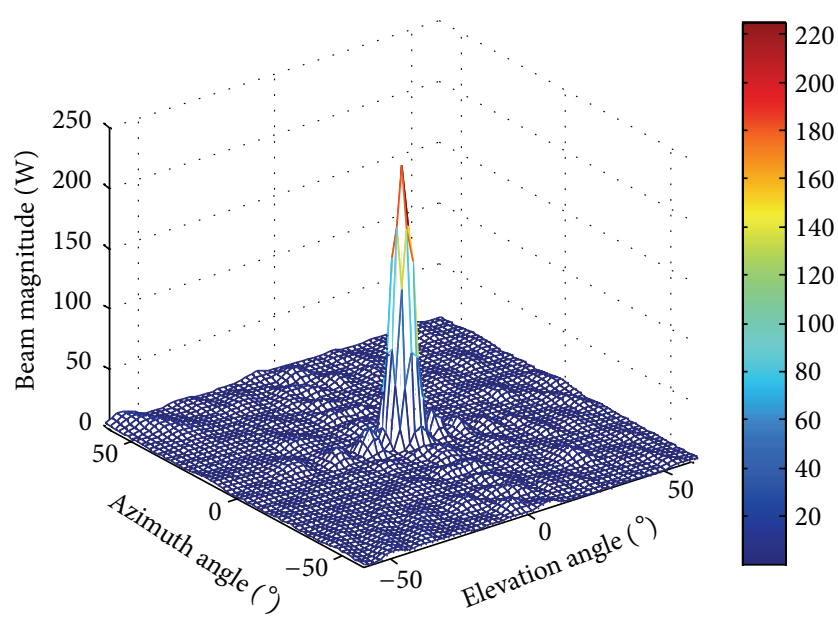

FiguRE 8: 3D beam patterns of power $225 \mathrm{~W}$.

\section{Conclusions}

In this paper, a Bi-MOWDO technique for multiobjective optimization based GRG is presented and used to design the emitted beamformer of the opportunistic array radar. The proposed algorithm presents excellent optimization performance over the multiobjective problem of optimization of beamforming and proves to outperform the Bi-MOWDO method.

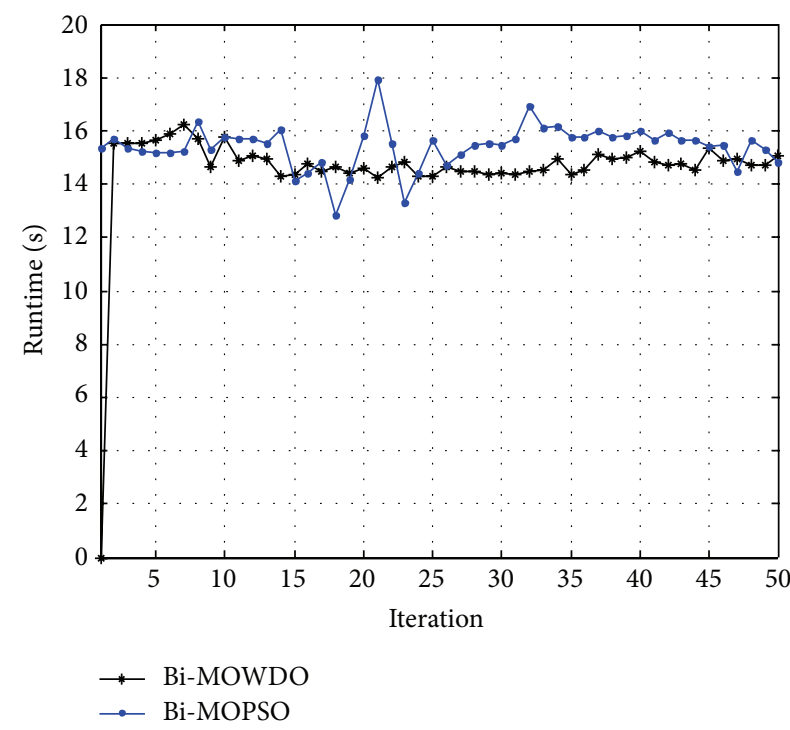

FIgURE 9: Runtime of every iteration for power $225 \mathrm{~W}$.

\section{Conflict of Interests}

The authors declare that there is no conflict of interests regarding the publication of this paper.

\section{Acknowledgments}

This work was supported by the National Natural Science Funds Fund (61401179), Colleges of Jiangsu Province Natural Science Fund (14KJB510009), the Science and Technology on Electronic Information Control Laboratory Project, Scientific Research Start-Up Funding from Jiangsu University of Science and Technology, Fundamental Research Funds for the Central Universities (NJ20140010), and a project funded by the Priority Academic Program Development of Jiangsu Higher Education Institutions.

\section{References}

[1] D. Jenn, Y. Loke, T. C. H. Matthew, Y. E. Choon, O. C. Siang, and Y.S. Yam, "Distributed phased arrays and wireless beamforming networks," International Journal of Distributed Sensor Networks, vol. 5, no. 4, pp. 283-302, 2009.

[2] W.-J. Long, D. Ben, M.-H. Pan, X.-R. Shu, Y.-M. Han, and J.B. Pan, "Opportunistic Digital Array Radar and its technical characteristic analysis," in Proceedings of the IET International Radar Conference, pp. 1-4, Guilin, China, April 2009.

[3] S. Gong, W. Long, H. Huang, D. Ben, and M. Pan, "Polyphase orthogonal sequences design for opportunistic array radar via HGA," Journal of Systems Engineering and Electronics, vol. 24, no. 1, pp. 60-67, 2013.

[4] K. Sindhya, K. Miettinen, and K. Deb, "A hybrid framework for evolutionary multi-objective optimization," IEEE Transactions on Evolutionary Computation, vol. 17, no. 4, pp. 495-511, 2013.

[5] B. Xue, M. Zhang, and W. N. Browne, "Particle swarm optimization for feature selection in classification: a multi-objective approach," IEEE Transactions on Cybernetics, vol. 43, no. 6, pp. 1656-1671, 2013. 
[6] S. Ganguly, "Multi-objective planning for reactive power compensation of radial distribution networks with unified power quality conditioner allocation using particle swarm optimization," IEEE Transactions on Power Systems, vol. 29, no. 4, pp. 1801-1810, 2014.

[7] J. Lu, D. Ireland, and A. Lewis, "Multi-objective optimization in high frequency electromagnetics - an effective technique for smart mobile terminal antenna (SMTA) design," IEEE Transactions on Magnetics, vol. 45, no. 3, pp. 1072-1075, 2009.

[8] A. Soroudi and M. Afrasiab, "Binary PSO-based dynamic multiobjective model for distributed generation planning under uncertainty," IET Renewable Power Generation, vol. 6, no. 2, pp. 67-78, 2012.

[9] Z. Bayraktar, M. Komurcu, J. A. Bossard, and D. H. Werner, "The wind driven optimization technique and its application in electromagnetics," IEEE Transactions on Antennas and Propagation, vol. 61, no. 5, pp. 2745-2757, 2013.

[10] X. Li, L. D. Li, and X. Yu, "Power generation loading optimization using a multi-objective constraint-handling method via PSO algorithm," in Proceddings of the 6th IEEE International Conference on Industrial Informatics (IEEE INDIN '08), pp. 1632-1637, Daejeon, korea, July 2008.

[11] K. Sindhya, K. Miettinen, and K. Deb, "A hybrid framework for evolutionary multi-objective optimization," IEEE Transactions on Evolutionary Computation, vol. 17, no. 4, pp. 495-511, 2013.

[12] D. Ju-Long, "Control problem of grey systems," Systems \& Control Letters, vol. 1, no. 5, pp. 288-294, 1982.

[13] H.-F. Ke, S.-F. Liu, Y.-G. Chen, and Z.-G. Fang, "New solution algorithm for multiple objective programming model based on grey relational degree," Systems Engineering and Electronics, vol. 32, no. 3, pp. 544-547, 2010. 

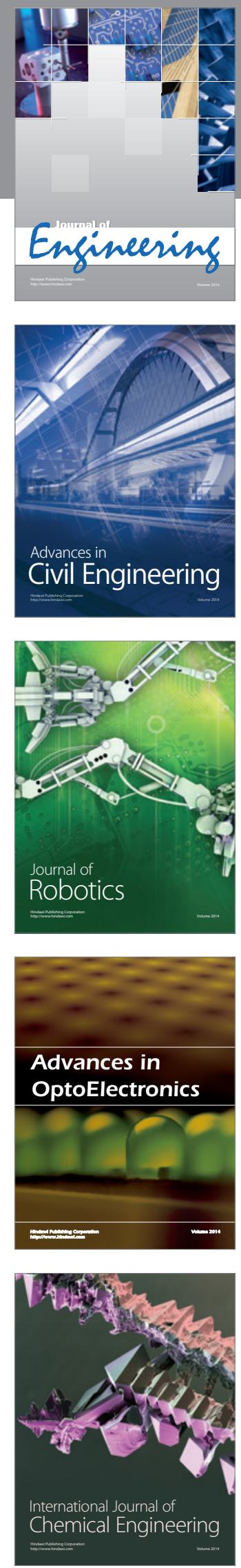

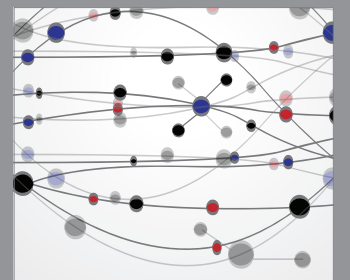

The Scientific World Journal
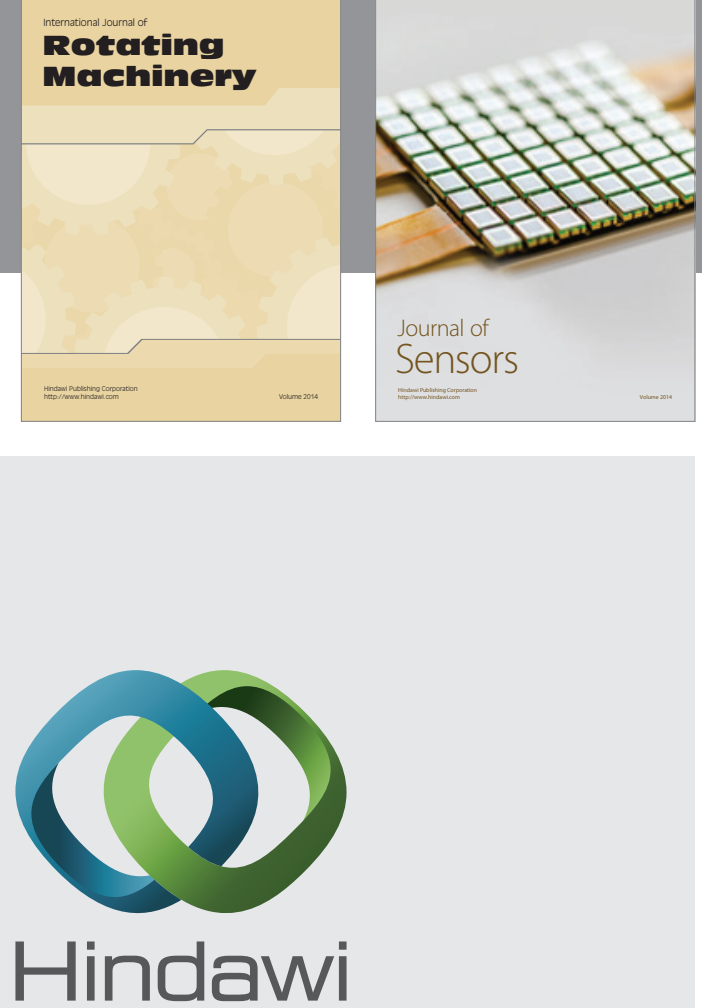

Submit your manuscripts at http://www.hindawi.com
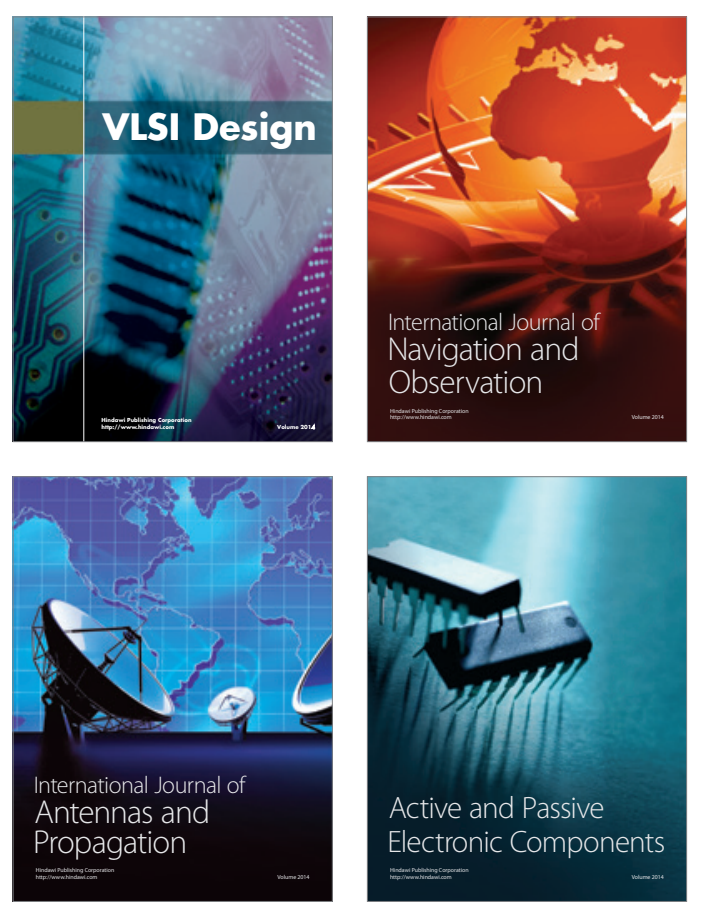


Journal of

Control Science

and Engineering
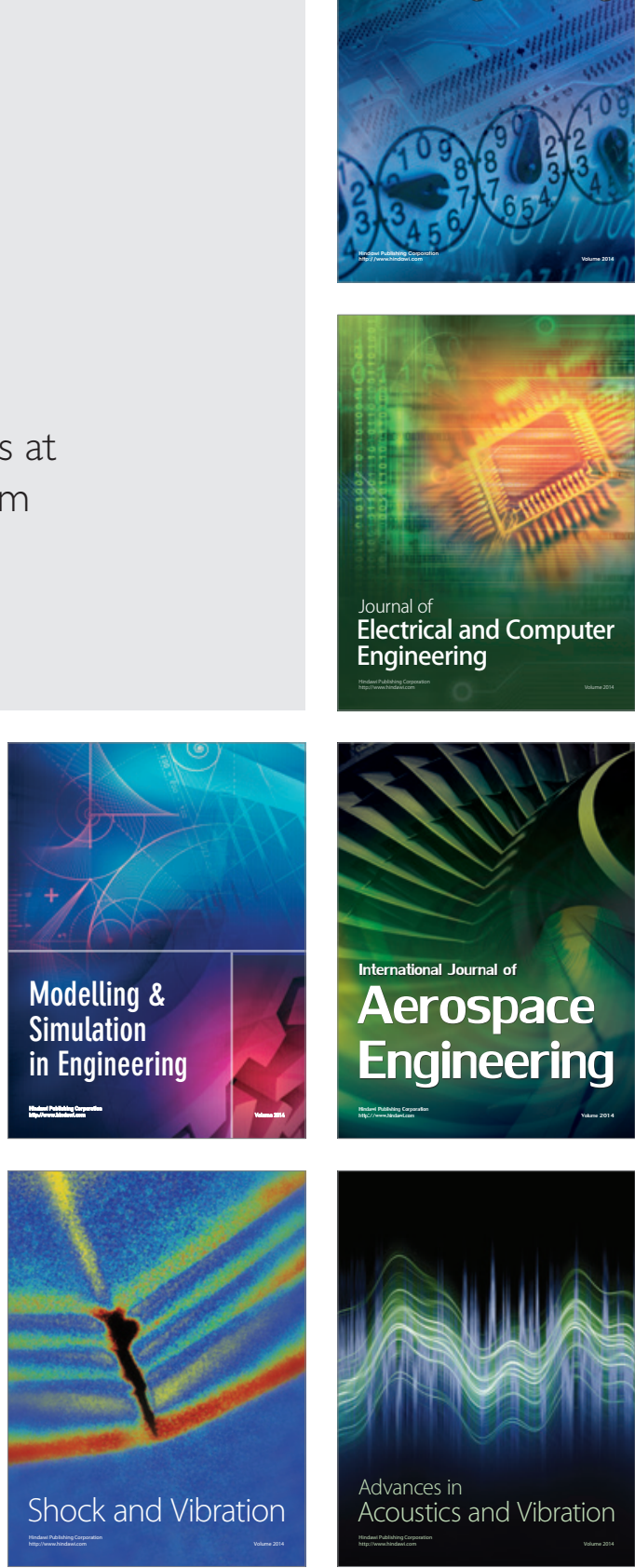